\title{
Caracterización espacio-temporal del hábitat y presencia de Dermatemys mawii (Testudines: Dermatemydidae) en la cuenca del Grijalva-Usumacinta, Tabasco, México
}

\author{
Claudia Elena Zenteno Ruiz ${ }^{1}$, Everardo Barba Macias² ${ }^{2}$ Joaquín Bello-Gutiérrez ${ }^{1}$ \\ \& Susana Ochoa-Gaona ${ }^{2}$ \\ 1. División Académica de Ciencias Biológicas. Universidad Juárez Autónoma de Tabasco. Carretera Villahermosa- \\ Cárdenas km. 0.5. Entronque Bosques de Saloya. C.P. 86150. Villahermosa, Tabasco; cezenteno@yahoo.com \\ 2. El Colegio de la Frontera Sur. Carretera Villahermosa/Reforma Km 15.5 Ranchería Guineo 2a. Sección. Villahermosa, \\ Tabasco. CP 86280; ebarba@ecosur.mx
}

Recibido 21-IX-2009. Corregido 03-V-2010. Aceptado 08-VI-2010.

\begin{abstract}
Presence and spatio-temporal habitat characterization of Dermatemys mawii (Testudines: Dermatemydidae) in the Grijalva-Usumacinta watershed, Tabasco, Mexico. The Central American River Turtle (Dermatemys mawii) is an endangered species that has been poorly studied. There are no reports on their population status, habitat condition, and the species distribution area is still unknown. This study analyzes the seasonal and spatial variations of their habitat and the presence/absence of $D$. mawii in three rivers within the Pantanos de Centla Biosphere Reserve (Tabasco, Mexico). For habitat characterization, natural segmentation of rivers was used and three sites per segment were identified, 9 in each rivers (Grijalva and Usumacinta) and 6 in Tabasquillo. Additionally, the evaluation of 11 environmental variables such as water hydrological, physicochemical characteristics and riparian and hydrophytic vegetation were carried out during two different seasons (dry and rainy). The presence/absence of species was assessed with eight fike nets that were set per segment, with a capture effort of 384 hours per trap. The capture per unit effort (CPUE) was used as an indicator of relative abundance. The results indicated spatio-temporal variations in habitat characteristics and the presence of environmental gradients. The principal components analysis (PCA) applied allowed us to determine that the first three components explained $67.8 \%$ of the environmental variability. The species presence was confirmed in all rivers, however significant differences exists in their relative abundance: the highest was registered in the Tabasquillo River where the species was present in both seasons and in all segments. Of the 11 environmental variables analyzed, the gradient, shelter and depth were the most indicative of species presence. The obtained results evidenced the importance of riparian vegetation as habitat for Dermatemys. This represents the first approach towards an action plan for a species and its habitat protection within the Pantanos de Centla Biosphere Reserve. Rev. Biol. Trop. 58 (4): 1247-1260. Epub 2010 December 01.
\end{abstract}

Key words: turtle conservation, lotic habitat, relative abundance, Pantanos de Centla.

La tortuga blanca Dermatemys mawii (Gray 1847) es una de las 25 tortugas en mayor riesgo de desaparecer a nivel mundial, única representante viviente de la Familia Dermatemydidae y la tortuga dulceacuícola más grande de México (Turtle Conservation Fund 2002, Vogt et al. 2006). En el 2006, la Unión Internacional para la Conservación de la Naturaleza la ubicó en la categoría de «Críticamente Amenazada», lo cual indica que está enfrentando un riesgo extremadamente alto de extinción; ubicándose como la tortuga mesoamericana con mayor riesgo de desaparecer en un futuro inmediato (UICN 2009, Vogt et al. 2006). En México, está considerada como una especie en Peligro de Extinción según la NOM-059-SEMARNAT-2001 debido a que su área de distribución y el tamaño de sus poblaciones en el territorio 
nacional han disminuido drásticamente poniendo en riesgo su viabilidad biológica en todo su hábitat natural, además es evidente su uso por las comunidades rurales en todo su rango de distribución (Polisar 1990). Su historia de vida se encuentra parcialmente estudiada (Moll 1986a, 1992, Polisar 1990, Vogt 1992, Vogt \& Flores-Villela 1992a, 1992b, Zenteno 1993, Moll \& Moll 2004) y se refiere a estudios taxonómicos o reportes sobre su presencia puntual en algunas localidades (Lee 1969, Álvarez del Toro et al. 1979, Flores-Villela 1980, Iverson \& Mittermeir 1980, Moll 1986a) y en menor proporción a características demográficas (Vogt 1992, Polisar 1995, 1996).

La Reserva de la Biosfera Pantanos de Centla (RBPC) es un humedal considerado como prioritario para la conservación de $D$. mawii (Vogt 1992). Está ubicada en un área de endemismo de tortugas neotropicales (Ippi \& Flores 2001), con características hidrológicas importantes como sitio de referencia para generar información sistemática sobre las variaciones espacio-temporales de la calidad del hábitat y estado de las poblaciones de D. mawii (CONABIO-DGVS-CONANP 2006), que permita conocer la capacidad del sistema y generar los recursos necesarios para satisfacer los requerimientos de vida de la especie (Moll \& Lagler 1971, Jones 1986, Moll \& Moll 2004). El presente trabajo se realizó en la cuenca baja de los ríos Grijalva, Usumacinta y Tabasquillo en la porción Norte de la Reserva de la Biosfera Pantanos de Centla, con el objetivo de analizar la variación espacio-temporal del hábitat en dos temporadas climáticas y confirmar la presencia de D. mawii y su abundancia relativa. Los resultados permitirán establecer las bases para definir áreas estratégicas para la protección y restauración del hábitat, monitoreo de las poblaciones remanentes y lograr una participación comunitaria, acciones prioritarias que se insertan en la estrategia nacional e interinstitucional para la protección de la especie en México.

\section{MATERIALES Y MÉTODOS}

El trabajo se realizó en la cuenca baja de los ríos Grijalva-Usumacinta, en la porción Norte de la RBPC en el estado de Tabasco, México, en un periodo anual (2005-2006). El área de estudio comprende parte del delta más importante de Norte y Centroamérica (INIREB 1986), donde los ríos Usumacinta y Grijalva discurren con volúmenes anuales de 27013 y 55832 millones de metros cúbicos de agua respectivamente (INE-SEMARNAP 2000).

El clima característico del área es Am(f) cálido húmedo con lluvias abundantes en verano, con una precipitación media anual entre $1500-2000 \mathrm{~mm}$ y variaciones a lo largo del año en la época fría y cálida (INEGI 2000). El régimen climático para 2005 presentó una menor precipitación durante los meses de febrero, marzo, abril y mayo, ausencia de precipitación en marzo y abril; los meses más lluviosos fueron agosto, septiembre y octubre. El promedio anual de temperatura fue de $25.1^{\circ} \mathrm{C}$, con un intervalo de 20.8 a $29.9^{\circ} \mathrm{C}$, siendo los meses de junio y julio los más calurosos.

La vegetación de la RBPC incluye nueve tipos de comunidades: 1) hidrófilas, 2) selva mediana subperennifolia de Bucidas buceras, 3) selva baja subperennifolia de Haematoxylum campechianum, 4) manglar, 5) matorral de Dalbergia brownei, 6) palmar de Acoelorraphe wrightii, 7) palmar de Sabal mexicana, 8) vegetación riparia dominada por Salix humboldtiana, Inga vera, Lonchocarpus hondurensis y Coccoloba barbadensis y 9) cultivos y potreros (INE 2000, Novelo 2006). La vegetación en la Reserva está sujeta a un proceso de continuo cambio y fragmentación, provocado principalmente por la deforestación e incendios forestales, especialmente los fragmentos de selva baja de $H$. campechianum y selva mediana de $B$. buceras presentan una importante tasa de disminución (Guerra-Martínez \& OchoaGaona 2006). 
Delimitación de los puntos de muestreo: Los sistemas hidrológicos en donde se realizaron los muestreos fueron los ríos Grijalva, Usumacinta y Tabasquillo. Previo al trabajo de campo se revisó la cartografía del área con el fin de identificar la segmentación (seg.) natural de los ríos para establecer los sitios de muestreo, los segmentos se verificaron en el campo mediante tres recorridos por el cauce de los ríos. En el Río Tabasquillo se delimitaron dos segmentos (TAB1 y TAB2), en el Grijalva y el Usumacinta tres (GRI1, GRI2 y GRI3; USU1, USU2 y USU3), respectivamente. En cada segmento se ubicaron tres sitios de repetición (rep.) para una mayor representatividad de la condición ambiental (Fig. 1), cubriendo dos temporadas climáticas (tem.): seca (S) y lluvia (LL) del año 2005. En total se ubicaron 24 sitios que se evaluaron en dos temporadas, con un total de 48 evaluaciones de hábitat, 18 en el Grijalva (3seg./3rep./2 tem.), 18 en el Usumacinta (3seg. /3rep./2tem.) y 12 en el Tabasquillo (2seg./3rep./2tem.).
Caracterización del hábitat: Debido a la escasa bibliografía sobre la relación especiehábitat, el conjunto de variables del hábitat se determinaron a partir de la consulta a siete investigadores con experiencia de campo sobre las condiciones de hábitat necesarias para la reproducción y desarrollo de D. mawii, para tal fin se utilizó la técnica Delphy (Crance 1987, Uhmann et al. 2001, Clevenger et al. 2002) y como resultado se establecieron 11 variables, de las cuales tres correspondieron a características hidrológicas, tres a condiciones fisicoquímicas del agua y cinco a vegetación.

Características hidrológicas. La profundidad (PROF) se determinó a $2 \mathrm{~m}$ de la orilla del río y se utilizó el disco de Secchi con una cuerda graduada cada $5 \mathrm{~cm}$; la velocidad (VEL) fue medida en la parte media del río a partir de la relación del tiempo que tardó en recorrer un objeto flotante un tramo de distancia conocida (20m); la pendiente del talud (PEND) se estimó a partir del porcentaje de inclinación entre los

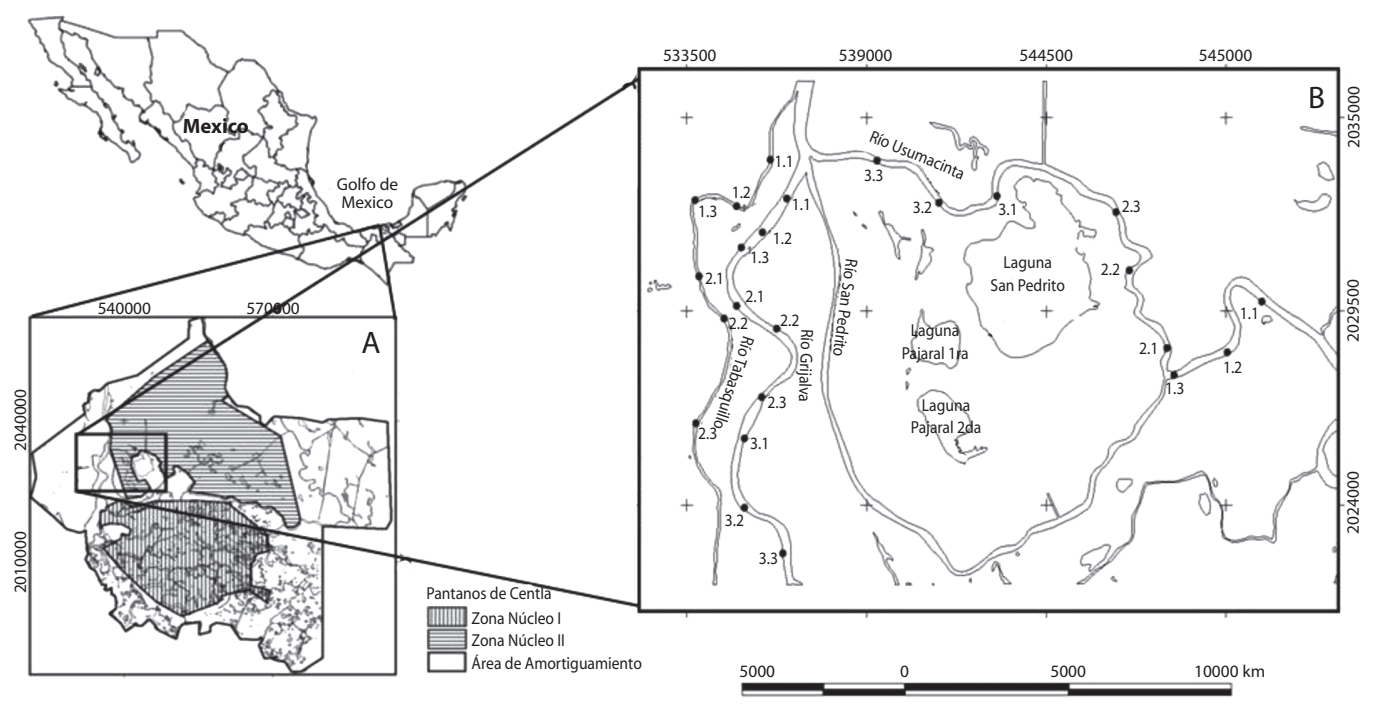

Fig. 1. Reserva de la Biosfera Pantanos de Centla (RBPC), Sureste de México, Estado de Tabasco (A) área de estudio, incluye la porción noroeste de la RBPC en la confluencia de los ríos Usumacinta-Grijalva-Tabasquillo (B) los números a un lado de los puntos indican el número de segmento y su repetición.

Fig. 1. The Pantanos de Centla Biosphere Reserve (RBPC) is located in the South Eastern part of Mexico, in the state of Tabasco (A).The study area includes the North Eastern portion of the RBPC in the confluence of the Usumacinta-GrijalvaTabasquillo rivers (B). The numbers next to the points indicate each segment and its repetition. 
puntos 0 y $4 \mathrm{~m}$ del borde del río dividido entre el respectivo cambio en las profundidades.

Características físico-químicas. La transparencia del agua (TRANS) se midió con el disco de Secchi; la temperatura (TEMP) se midió con un termómetro; el oxigeno disuelto (OXI) se midió con un oxímetro (Hanna ${ }^{\circledR}$ HI9143) y se expresó como el promedio entre la superficie y los $50 \mathrm{~cm}$ de profundidad.

Vegetación. La cobertura hidrófita (COBHIDR) se calculó sobre el cauce del río en la franja próxima al borde a partir de un cuadrante de $1 \mathrm{x} 1 \mathrm{~m}$ y se expresó como el porcentaje promedio de tres repeticiones; la riqueza hidrófita (RIQZHIDR) se determinó como el número promedio de especies presentes en los cuadrantes; la cobertura ribereña (COBRIP) a partir de una unidad de muestreo ubicada en el área terrestre paralela a los bordes de río y donde se calculó la cobertura total utilizando el método de intersección en línea (método de Canfield, Ojasti 2000). La riqueza ribereña (RIQZRIP) se determinó como el número de especies presentes en el transecto de $50 \times 4 \mathrm{~m}$ de ancho; el refugio (REF) se estimó en un cuadrante reticulado de 20x4m evaluándose el porcentaje de cobertura vegetal (ramas, troncos o raíces) sobre el espejo de agua (Segnini \& Chacon 2005). Se recolectaron 120 especimenes botánicos de vegetación hidrófila y ribereña conforme las técnicas de herborización convencionales. La identificación se realizó con apoyo del personal del herbario de la Universidad Juárez Autónoma de Tabasco.

\section{Presencia y abundancia relativa del} hábitat de D. mawii: Las capturas de organismos se realizaron en ambas temporadas (seca y lluviosa), en cada sistema hidrológico y segmento se colocaron ocho trampas de desvío acuáticas tipo Fike Net Set durante 48 horas (Graham 1979, Vogt 1980). La captura por unidad de esfuerzo (CPUE) se determinó como el número de organismos capturados por segmento dividido entre el número de trampas colocadas y se utilizó como indicador de la abundancia relativa. Los sitios de captura se ubicaron geográficamente mediante un sistema de posicionamiento global (GPS) para su posterior representación en el Sistema de Información Geográfica (SIG). Los organismos capturados en las trampas se midieron bajo las técnicas convencionales para tortugas (Vogt 1980). El sistema de marcaje utilizado fue el propuesto por Plummer (1979), mediante muescas en las escamas marginales. Todos los organismos se liberaron en la proximidad de los sitios de captura el último día de muestreo. Para cada captura se ubicó geográficamente la localización de la trampa y se asumió la presencia de la especie para todo el segmento, considerando que puede realizar movimientos hasta de $2.5 \mathrm{~km}$ por día (Vogt \& Flores-Villela 1992). Los rastros de actividad de la especie (ramoneo, anidación y heces) se identificaron con ayuda de un guía de campo y se georeferenciaron para establecer sitios de mayor actividad.

Para conocer si las características del hábitat variaban entre los ríos y temporadas, se aplicó un análisis de varianza no paramétrica de Kruskal-Wallis con un nivel de significancia de $\mathrm{p} \leq 0.05$ y para reducir la dimensionalidad de las variables y ordenación de los sitios, se realizó un análisis de componentes principales (ACP) empleando la matriz de correlaciones y rotación ortogonal Varimax, para el mejor ajuste e interpretación de las componentes. Los valores de captura por sistema y temporada se representaron sobre los dos primeros ejes con el fin de visualizar su relación con los gradientes detectados en el ACP.

Para determinar qué variables predicen la presencia de la especie se realizó un árbol de clasificación (CART por sus siglas en inglés; Breiman et al. 1984), el cual permitió identificar las relaciones no lineales entre la variable respuesta (presencia/ausencia de D. mawii) y los predictores (variables del hábitat), así como las interacciones entre predictores. Esta técnica no paramétrica divide recurrentemente el conjunto de datos en nodos. En cada instancia de separación el algoritmo analiza todas las variables predictoras y selecciona, para realizar la partición, aquella que permite conformar grupos más homogéneos internamente y más 
heterogéneos entre sí. El procedimiento no asume una distribución específica de los datos por lo que no requiere transformaciones y no es influido por valores extremos (Breiman et al. 1984, De'ath \& Fabricius 2000, Vayssie'res et al. 2000). Por tratarse de una variable de clasificación se empleó la devianza como medida de heterogeneidad dentro de cada nodo. Las pruebas estadísticas se realizaron en los programas JMP (ver. 7, SAS Institute), Infostat (ver. 1.1) y Past (ver 5.1, Statsoft inc.).

\section{RESULTADOS}

Características del hábitat: Las características del hábitat en los tres sistemas presentaron un amplio gradiente de condiciones en los sitios analizados. La profundidad varió de 0.25 a $>6.0 \mathrm{~m}$. El Usumacinta es el sistema de mayor profundidad; en la temporada de lluvias ésta se incrementa en todos los hábitats: Grijalva de 1.10 a $1.96 \mathrm{~m}$, Usumacinta de 1.57 a $2.62 \mathrm{~m}$ y Tabasquillo de 0.70 a $1.56 \mathrm{~m}$. La velocidad del agua mostró un rango de 0 a $1.05 \mathrm{~m} / \mathrm{s}$, observándose los valores medios más altos en los ríos Grijalva y Usumacinta; de una temporada a otra se incrementa la velocidad en los tres sistemas, siendo mayor en la época lluviosa. La pendiente del Grijalva y Usumacinta no difieren significativamente, mientras que Tabasquillo presenta un talud de pendiente más suave.

La transparencia del agua fluctuó entre 0.08 y $1.81 \mathrm{~m}$, observándose mayor transparencia en Tabasquillo; en la temporada de lluvias la transparencia disminuye en Grijalva y Usumacinta, mientras que en Tabasquillo el efecto es inverso. La temperatura varió entre 20.6 y $33^{\circ} \mathrm{C}$, en el Tabasquillo se registraron las temperaturas medias más altas y la mayor fluctuación entre temporadas. El oxígeno disuelto presentó un intervalo entre 0.44 y $9.12 \mathrm{mg} / 1$, las concentraciones disminuyeron en los tres sistemas durante la temporada de lluvia.

La cobertura hidrófita en el Usumacinta estuvo ausente en ambas temporadas, para los otros dos sistemas fluctuó entre el 0 y $100 \%$, en todos los puntos se registró un incremento durante la temporada lluviosa. La riqueza de especies hidrófilas encontradas en los cuadrantes fluctuó ente 1 y 5 especies, Tabasquillo presentó el mayor número de especies hidrófilas, siendo las más comunes: Eichhornia crassipes, Pistia stratiotes e Hydrocotyle umbelata. La cobertura ribereña presentó un rango entre 0 y $95.2 \%$, observándose la mayor cobertura en el Tabasquillo contrario a lo que ocurrió en el Usumacinta con valores menores al 50\%. La riqueza de especies ribereñas fluctuó entre 0 y 10 especies, en el Tabasquillo se registró el mayor número de especies, entre las más comunes están: S. mexicana, $H$. campechianum, $C$. barbadensis y Lonchocarpus sp. La cobertura de refugio varió entre 0 y $100 \%$, el Tabasquillo presentó los valores más altos en todos los tramos evaluados, el Grijalva presenta una condición intermedia y el Usumacinta fue el más desprotegido.

Los valores medios, desviación estándar y los resultados de Kruskal-Wallis se presentan en el Cuadro 1. Entre los tres ríos se dieron diferencias espaciales significativas en las variables de velocidad, transparencia, cobertura hidrófita, riqueza hidrófita, cobertura ribereña, riqueza ribereña y refugio. Se presentaron diferencias temporales en la profundidad, velocidad, transparencia, temperatura y oxígeno disuelto. La pendiente fue la única variable que no presentó diferencias significativas temporales o espaciales.

El análisis de componentes principales mostró que los tres primeros ejes explican el $67.83 \%$ de la varianza total, con una separación clara de los tres tipos de variables ambientales caracterizados. El primer eje (CP1) explica el $34.04 \%$ de la varianza y representa un gradiente a través de las variables de vegetación (COBHIDR, RIQHID Y REF). El segundo eje (CP2) se relaciona con variables físicoquímicas del agua (TEMP Y OXI) explicando el $20.6 \%$ de la varianza. El tercer eje (CP3) explica el $13.12 \%$ de la varianza y está asociado con las variables hidrológicas (PROF Y PEND) (Cuadro 2).

El ordenamiento de los 48 segmentos muestreados en CP1 y CP2 (Fig. 2), permitió observar la agregación o dispersión de los 


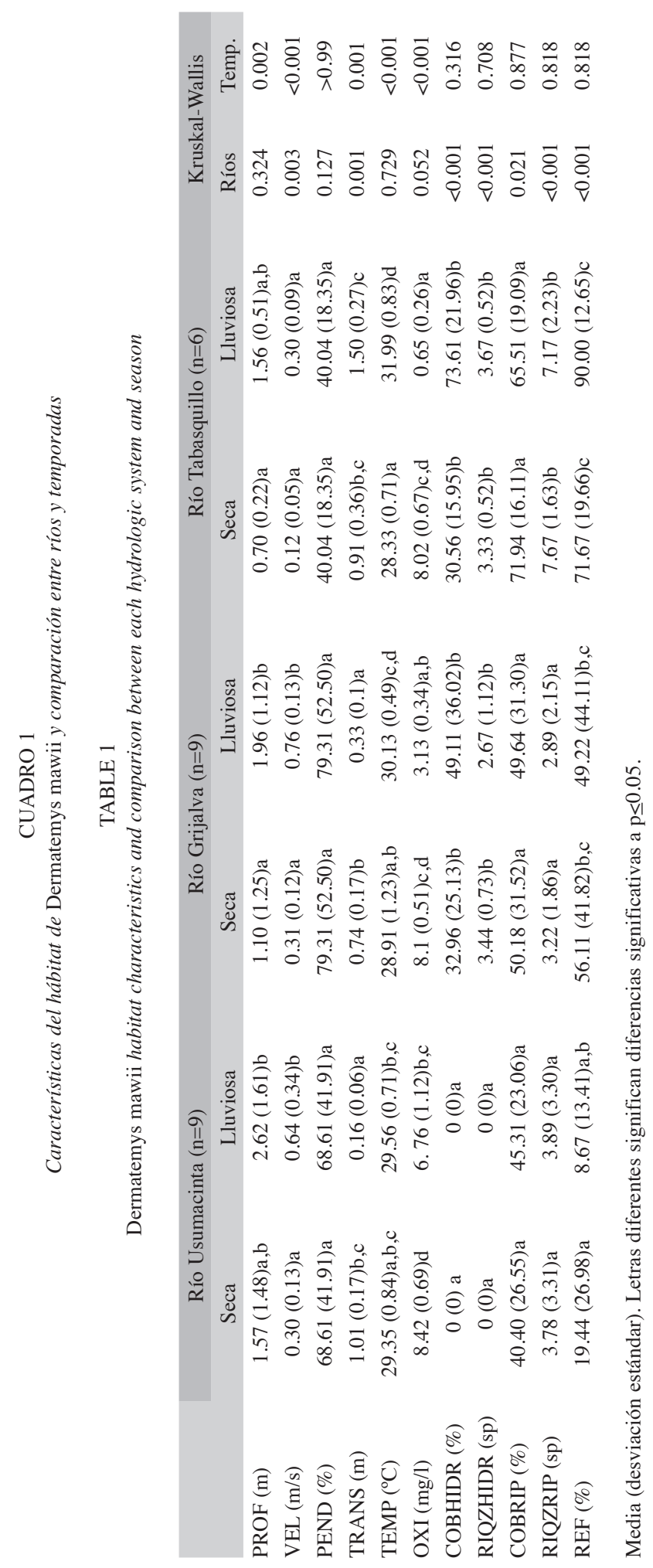


CUADRO 2

Peso de los factores y varianza explicados en el análisis de componentes principales del hábitat en tres ríos de la Reserva de la Biosfera Pantanos de Centla

TABLE 2

Factor loadings and variance explained from a principal components analysis on habitat variables sampled within three rivers in Pantanos de Centla Biosphere Reserve

\begin{tabular}{|c|c|c|c|c|c|}
\hline & $\mathrm{CP} 1$ & $\mathrm{CP} 2$ & $\mathrm{CP} 3$ & CP4 & CP5 \\
\hline \multicolumn{6}{|l|}{ Variables } \\
\hline \multicolumn{6}{|l|}{ Hidrológicas } \\
\hline PROF (m) & -0.253 & 0.163 & 0.906 & 0.085 & -0.122 \\
\hline $\operatorname{VEL}(\mathrm{m} / \mathrm{s})$ & -0.091 & 0.329 & 0.527 & -0.176 & -0.681 \\
\hline PEND $(\%)$ & 0.113 & -0.231 & 0.890 & -0.249 & -0.025 \\
\hline \multicolumn{6}{|l|}{ Físico-químicas } \\
\hline TRANS (m) & 0.232 & 0.174 & -0.013 & 0.094 & 0.918 \\
\hline TEMP $\left({ }^{\circ} \mathrm{C}\right)$ & 0.035 & 0.920 & -0.071 & -0.062 & 0.108 \\
\hline OXI (mg/l) & -0.362 & -0.855 & -0.096 & -0.130 & 0.086 \\
\hline \multicolumn{6}{|l|}{ Vegetación } \\
\hline COBHIDR (\%) & 0.829 & 0.394 & -0.088 & 0.069 & 0.047 \\
\hline RIQZHIDR (sp) & 0.898 & 0.048 & -0.089 & 0.109 & 0.167 \\
\hline COBRIP $(\%)$ & 0.325 & 0.017 & -0.119 & 0.816 & -0.112 \\
\hline RIQZRIP (sp) & 0.073 & 0.015 & -0.055 & 0.830 & 0.326 \\
\hline $\operatorname{REF}(\%)$ & 0.772 & 0.042 & 0.005 & 0.421 & 0.179 \\
\hline Varianza explicada (\%) & 34.044 & 20.664 & 13.125 & 9.889 & 9.249 \\
\hline Varianza acumulada (\%) & 34.044 & 54.707 & 67.833 & 77.722 & 86.971 \\
\hline
\end{tabular}

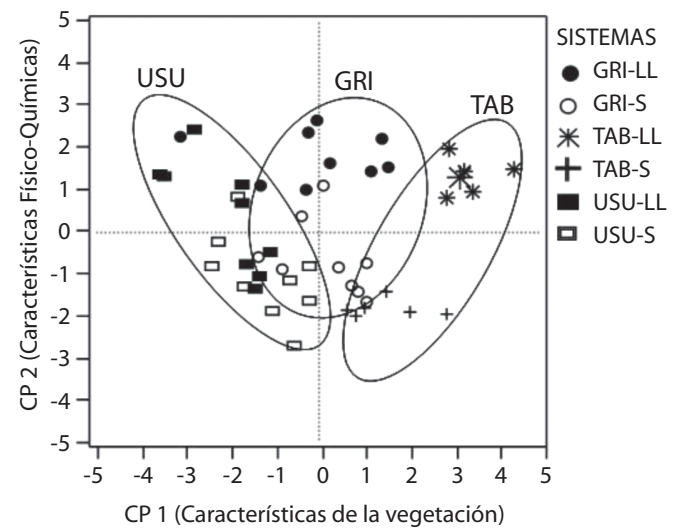

Fig. 2. Ordenamiento de los segmentos de los ríos en relación con los CP1 y CP2: GRI (Río Grijalva), TAB (Río Tabasquillo) y USU (Río Usumacinta) en temporada de lluvia (LL) y seca (S).

Fig. 2. Ordination of the segments of the rivers in relation to the CP1 and CP2: GRI (Grijalva River), TAB (Tabasquillo River) and USU (Usumacinta River) in the rainy season (LL) and dry season (S). segmentos por sistema en los gradientes de la gráfica. Sobre el CP1 se ubican los segmentos del Tabasquillo hacia valores positivos más altos, es decir están caracterizados por una mayor cobertura hidrófita y de refugio, además que sus segmentos están próximos entre sí, lo que indica que son relativamente homogéneos, pero difieren temporalmente. En el extremo contrario se ubican los segmentos del Usumacinta con los valores más bajos y mayor heterogeneidad de sus segmentos (dispersión de los segmentos sobre el plano). La condición intermedia del gradiente la ocupó el Río Grijalva. La dispersión de los segmentos sobre el CP2 indica una relativa diferenciación de los segmentos en función de la temporalidad.

Presencia, abundancia relativa y relación con las variables del hábitat de $D$. mawii: Durante la época seca se capturaron 
seis ejemplares, cinco en Tabasquillo (0.312 ind/trampa), uno en Grijalva (0.041 ind/trampa) y ninguno en Usumacinta. Durante la época lluviosa se capturaron once individuos, ocho en Tabasquillo (0.500 ind/trampa), dos en Grijalva (0.083 ind/trampa) y uno en Usumacinta (0.041 ind/trampa). No se encontraron diferencias significativas atribuidas a las temporadas $(\mathrm{H}=0.29$, $\mathrm{n}=48, \mathrm{p}=0.44$ ), pero si existieron diferencias entre los ríos $(\mathrm{H}=5.57, \mathrm{n}=48, \mathrm{p}<0.05)$, encontrándose una mayor abundancia relativa en el Tabasquillo donde la especie estuvo presente en ambas temporadas y en todos los segmentos muestreados.

El árbol de clasificación se construyó con las 11 variables predictoras, de las cuales cuatro tuvieron mayor peso para integrar el modelo (Fig. 3). El árbol presenta un nodo raíz que incluye las variables de los 48 sitios, la primera partición que ajusta al modelo es la pendiente (PEN) que divide al árbol en dos ramas, la primera indica que cuando la pendiente es mayor o igual a $84.5 \%$ la especie está ausente. En condiciones de pendientes más suaves $(>84.5 \%)$ y si la condición del refugio (REF) es mayor a $80 \%$ hay una probabilidad de 1 de encontrar la especie. Con valores de refugio menores, la presencia de la especie está relacionada con la cobertura de la vegetación hidrófila (COBHID) en donde el $19.3 \%$ es valor de partición, valores mayores indican el 0.625 de probabilidad de encontrar la especie y valores menores indican que están condicionadas a la partición final correspondiente a la variable profundidad (PROF), con valores menores a $1.6 \mathrm{~m}$ la especie está ausente y valores mayores representan 0.200 de probabilidad de encontrarla.

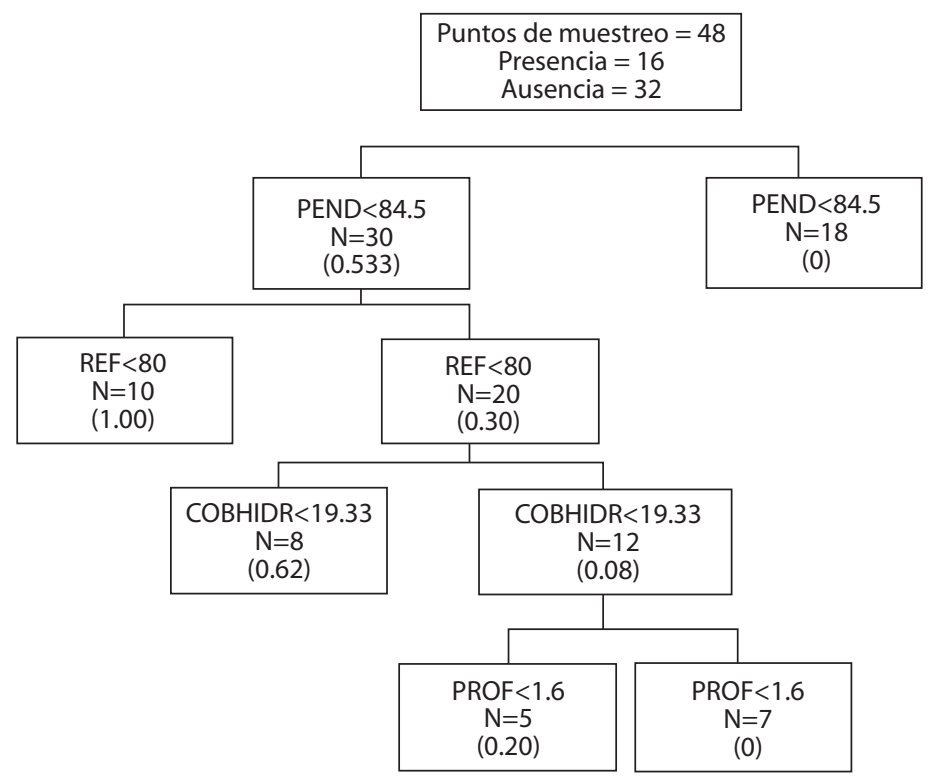

Fig. 3. Resultados del árbol de clasificación sobre la presencia de Dermatemys mawii y sus variables predictoras. Entre paréntesis la probabilidad de presencia por nodo.

Fig. 3. Classification tree results on the presence of Dermatemys mawii and the predictor variables of the habitat. The probabilities to presence are given in parentheses. 


\section{DISCUSIÓN}

Caracterización del hábitat: El análisis general de las características físico-bióticas del hábitat indicó que el área presenta una heterogeneidad espacial, con mayores contrastes entre Usumacinta y Tabasquillo, representando el Grijalva un sistema de condiciones intermedias. Estos gradientes ambientales son explicados por las diferencias en el origen de las cuencas, el Grijalva (que incluye la subcuenca del Tabasquillo) discurre sobre formaciones sedimentarias, quebradizas y de fácil erosión, así como sobre granitos gastados; mientras que la corriente del Usumacinta desagua en áreas de rocas calizas con altos contenidos de bicarbonato de calcio y sodio (West et al. 1985, Velázquez 1994). Ambas cuencas conforman un escurrimiento importante que representa el $30 \%$ del total nacional (INEGI 2000) y están asociadas con problemas de modificación de origen antrópico y natural, en particular el Grijalva que ha sido interrumpido por obras viales e hidráulicas (Tudela 1992).

Las diferencias hidromorfológicas de los ríos se relacionan con la frecuencia y tamaño de las inundaciones, que determinan la capacidad de las corrientes (Benda et al. 1998). En los tramos analizados es posible detectar diferencias en la naturaleza de las dos subcuencas, tales como la presencia de numerosos asentamientos, la expansión de la frontera agrícola y la actividad industrial, principalmente en el Usumacinta (Sánchez et al. 2007), lo que ha impactado principalmente la calidad del agua por el constante aporte de desechos domésticos (orgánicos) y residuos de las actividades agropecuarias, así como por la disminución de la vegetación ribereña y erosión de los bordes en ambos ríos. Dichos procesos tienen efectos directos sobre características fisicoquímicas del hábitat tales como: la velocidad, el oxígeno disuelto, la temperatura y transparencia del agua (Welch et al. 1998), que se consideran describen el hábitat de Dermatemys, han sido asociadas con la presencia de la especie en otras localidades (Álvarez del Toro et al. 1979, Vogt 1992, Polisar 1995, 1996).
Las diferencias entre los sistemas y gradientes ambientales detectados en la vegetación ribereña y acuática indican que existen condiciones heterogéneas de hábitat y salud del ecosistema (Jones 1986), que cubren en mayor o menor calidad los requerimientos de la especie (Moll \& Moll 2004).

Los principales cambios temporales sobre las características del hábitat están relacionados con las variables hidrológicas y físico-químicas (profundidad, velocidad, transparencia, temperatura y oxígeno disuelto), como resultado del aumento en el volumen de escurrimientos en las partes altas de ambas cuencas, así como del incremento en el aporte de materia en suspensión de origen alóctono y autóctono, que ocasiona variaciones en la transparencia y el oxígeno disuelto (Sánchez et al. 2007).

La cobertura de la vegetación hidrófita se incrementó en algunos segmentos, condición inestable dado que en su mayoría son especies libres flotadoras que no están fijas al sustrato. En el ambiente ribereño (cobertura ribereña, riqueza ribereña y refugio) no se registraron cambios temporales con significancia estadística, sin embargo, en algunos tramos se evidenció la tala de especies arbóreas para el aprovechamiento de madera, lo que ocasiona variaciones en la cobertura de la vegetación ribereña y modificaciones estructurales de las ramas que caen al cuerpo de agua y que constituyen parte del refugio de la especie.

Durante la época de seca los tres ríos están sujetos a la influencia del ambiente marino (Velazquez 1994), el efecto sobre el Tabasquillo es potencialmente mayor que para Usumacinta y Grijalva, debido a su menor caudal, condición que afecta la vitalidad de la vegetación hidrófita (Brock et al. 2005) y provoca diferencias ambientales entre temporadas, además que al aumentar la profundidad y cobertura de los cuerpos de agua en la época de lluvias se establecen vías de acceso a recursos disponibles en la llanura aluvial, como está demostrado en otras especies de tortugas dulceacuícolas (Moll \& Lagler 1971, Bodie \& Semlitsch 2000, Bodie et al. 2000, Moll \& Moll 2004). 
Presencia y abundancia relativa de la especie: La presencia de D. mawii se confirmó en todos los ríos estudiados: Usumacinta, Grijalva y Tabasquillo, siendo este último en donde la especie fue más abundante. Las condiciones del hábitat son mejores en el Tabasquillo como lo confirmaron los datos sobre la CPUE y presencia de la especie, en ambas temporadas (Cuadro 3). Las variables predictoras de la presencia de la especie están más relacionadas con las características de la estructura de la vegetación e hidromorfología que con las variables físico-químicas del agua.

La abundancia de D. mawii a través de su área de distribución es poco conocida. Hasta hace tres décadas en Belice se consideraba abundante y común: Moll (1986b, 1989) identificó una población con una densidad de 2.3ind/ ha en la Laguna Progreso, posteriormente Polisar (1995) utilizando diferentes métodos capturó 5.33tortugas/día mediante buceo y 1.82tortugas/red/día con el uso de redes de nylon de $12.6 \mathrm{~cm}$ de luz de malla. Datos empíricos actuales indican que las poblaciones en Belice son más grandes que las de México, pero es evidente la declinación de las poblaciones en todas las localidades conocidas de captura de la especie (Vogt et al. 2006).

En México los datos de abundancia son aún mas escasos, los únicos datos sistemáticos y documentados para el sureste de México son los obtenidos por Vogt \& Flores-Villela (1992b) en el Río Tzendales, Chiapas, donde capturaron 14 individuos con un índice de captura de $0.031 \mathrm{ind} /$ trampa; posteriormente en el 2002, Guichard (2006) realizaron el mismo esquema en las mismas localidades y capturaron un solo individuo con un índice de captura de 0.006 ind/trampa, es decir, disminuyó más del $80 \%$ en tan sólo 10 años. Esta es la única área para la que se tiene información de varios años.

Los índices de captura obtenidos en el presente trabajo son superiores a los reportados por Vogt \& Flores-Villela (1992b) y Guichard (2006), resultado de un muestreo con mayor cobertura espacial y temporal, así como una mayor interacción y trabajo colaborativo con informantes claves del área. Las evidencias indirectas (niveles de extracción y rastros) sobre las estimaciones de las poblaciones de tortugas, resultan insuficientes para establecer tendencias poblacionales debido a que las cuantificaciones han sido a partir de datos aislados, a la inconsistencia y a la falta de estandarización en los métodos y esfuerzos de captura (CONABIO-DGVS-CONANP 2006).

Las características de PEND, COBHIDR y COBRIP se confirman como variables predictoras de la especie e importantes para la alimentación y anidación de la especie (Álvarez del Toro et al. 1979, Polisar 1995, 1996, Moll \& Moll 2004), lo anterior se explica con la dinámica de inundación y la altura de los taludes. En el Tabasquillo es más probable el

CUADRO 3

Captura por unidad de esfuerzo (CPUE) de Dermatemys mawii en cada sistema

TABLE 3

Catch per unit effort (CPUE) of Dermatemys mawii in each system

\begin{tabular}{lcccccc}
\hline Temporada & Sistema & Hembra & Macho & Cría sin sexar & Total & CPUE \\
Seca & GRI & 1 & 0 & 0 & 1 & 0.041 \\
& TAB & 1 & 3 & 1 & 5 & 0.312 \\
\multirow{3}{*}{ Lluvia } & USU & 0 & 0 & 0 & 0 & 0.000 \\
& GRI & 2 & 0 & 0 & 2 & 0.083 \\
& TAB & 5 & 3 & 0 & 8 & 0.500 \\
& USU & 1 & 0 & 0 & 1 & 0.041
\end{tabular}

*La CPUE se determinó dividiendo la captura por sistema/número de trampas (16 trampas en TAB, 24 en GRI y 24 USU) 
desplazamiento de la hembra para la anidación, lo que coincide con el registro de rastros de nidos. Otras condiciones relacionadas con la construcción del nido, son los niveles de humedad del sustrato (Morjan \& Janzen 2003) y el entorno microclimático, favorables para mantener un equilibrio natural de sexos en $D$. mawii a través de la temperatura de incubación (Vogt \& Flores-Villela 1986). Las franjas continuas de vegetación ribereña proveen de refugio contra depredación de los huevos o hembras, debido a que disminuyen el efecto de borde (Moll 1986b, Kolbe \& Janzen 2002, Doody et al. 2004).

Uno de los aspectos notables a escala de paisaje, es que el río Tabasquillo está rodeado por una zona arbórea que le brinda un estado de conservación natural al sistema, condición importante para mantener poblaciones silvestres de tortugas (Joyal et al. 2001). Gran parte de los recursos alimenticios y estructuras que sirven como refugio provienen del ambiente ribereño (Semlitsch \& Bodie 2003), existiendo una relación positiva entre la cobertura vegetal y la estructura de las poblaciones de tortugas (Marchand \& Litvaitis 2004). La transformación y reducción del hábitat ribereño debido a la canalización, contaminación, cambio de uso del suelo y otros factores, tiene efectos negativos sobre las poblaciones de tortugas; ocasionando la pérdida de sitios de anidación, fragmentando las poblaciones, modificando la estructura o proporción de sexos y fomentando la extinción local (Bodie 2001). Lo anterior concuerda con los resultados del árbol de clasificación que indican una alta probabilidad de presencia de la especie en sitios con coberturas REF superiores al $80 \%$.

Con los resultados obtenidos, es posible fundamentar las bases para un plan de acción en la RBPC, el cual debe incluir el monitoreo y manejo adaptativo del hábitat crítico y poblaciones remanentes, así como la integración de las comunidades humanas a la conservación de la especie.

\section{AGRADECIMIENTOS}

Este trabajo fue financiado por los Fondos sectoriales SEMARNAT-CONACYT mediante el proyecto "Evaluación del estado de Conservación del hábitat de Dermatemys mawii en la Reserva de la Biosfera Pantanos de Centla". Agradecemos a la Dirección de la RBPC las facilidades para la realización de la fase de campo; al Dr. Richard Vogt por proporcionar equipo para las capturas y a la Cooperativa "Arroyo Tabasquillo" por las facilidades proporcionadas para realizar el trabajo en el río Tabasquillo. Al Ecol. Joaquín Hernández, M.C. Luis Felipe Zamora Cornelio y al C. Alcides Sánchez Pérez quienes colaboraron en el trabajo campo.

\section{RESUMEN}

La tortuga centroamericana (Dermatemys mawii) es una especie en peligro de extinción pobremente estudiada. En el presente trabajo se analizaron las variaciones estacionales y espaciales del hábitat y se relacionaron con la presencia/ausencia de D. mawii en tres ríos de la Reserva de la Biosfera Pantanos de Centla (Tabasco, México). Para caracterizar el hábitat se evaluaron 11 variables (hidrológicas, fisicoquímicas del agua y de la vegetación) en dos temporadas (seca y lluviosa). Para determinar la presencia/ ausencia de la especie se colocaron 8 trampas de desvío acuáticas, empleando la captura por unidad de esfuerzo (CPUE) como indicador de la abundancia relativa. Los resultados indicaron variaciones espacio-temporales. El análisis de componentes principales (ACP) permitió determinar la variabilidad ambiental. La presencia de la especie se confirmó en los tres ríos, sin embargo la mayor abundancia relativa se registró en el Río Tabasquillo. Cuatro variables tuvieron el mayor peso como variables predictoras de la presencia de la especie. Con los resultados obtenidos, es evidente la importancia que tiene el ambiente ribereño como hábitat para Dermatemys, asimismo es posible hacer el primer acercamiento a un plan de acción para la protección de la especie y su hábitat en esta reserva.

Palabras clave: Conservación de tortugas, hábitat lóticos, abundancia relativa, Pantanos de Centla.

\section{REFERENCIAS}

Álvarez Del Toro, M., R.A. Mittermeier \& J.B. Iverson. 1979. River turtle in danger. Oryx 15: 170-173. 
Benda, L., J. Millar DT., Dunne H., Reeves G. \& J.L. Agee. 1998. Dynamic Landscape System, p. 261-288. In E.B. Welch, J.M. Jacoby \& C.W. May (eds.). River Ecology and management. Lessons from the Pacific Coastal. Springer, Nueva York, EEUU.

Bodie, J.R. 2001. Stream and riparian management for freshwater turtles. Environ. Manage. 62: 443-455.

Bodie, J.R. \& R.D. Semlitsch. 2000. Spatial and temporal use floodplain habitat by lentic and lotic species of aquatic turtles. Oecologia 122: 138-146.

Bodie, J.R., R.D. Semlitsch \& R.B. Renken. 2000. Diversity and structure of turtle assemblages: associations with wetland characters across a floodplain landscape. Ecography 23: 444-456.

Breiman, L., J.H. Friedman \& R.A. Olsen. 1984.Classification and regression trees. Wadsworth International Group, Belmont, California, EEUU.

Brock, M.A., D.L. Nielsen \& K. Crosslé. 2005. Changes in biotic communities developing from freshwater wetland sediments under experimental salinity and water regimes. Freshwat. Biol. 50: 1376-1390.

Clevenger, A., J. Wierzchowsky, B. Chrustzcz \& K. Gunzon. 2002. Gis-Generated, Expert-based models for identifying wildlife habitat linkage and planning mitigation passage. Conserv. Biol. 16: 503-514.

CONABIO-DGVS-CONANP. 2006. Estrategia Nacional para la Conservación y el Manejo Sostenible de la Tortuga Blanca (Dermatemys mawii) en México. Comisión Nacional para el Conocimiento y Uso de la Biodiversidad, México, D.F., México.

Crance, J.H. 1987. Guidelines for using the Delphy technique to develop habitat suitability index curve. U.S. Fish Wildlife Serv. Biol. Rep. 82: 1-23.

De'ath, G. \& K.E. Fabricius. 2000. Classification and regression trees: a powerful yet simple technique for ecological data analysis. Ecology 81: 3178-3192.

Doody, J.S., A. Georges \& J.E. Young. 2004. Determinants of reproductive success and offspring sex in a turtle with environmental sex determination. Biol. J. Linn. Soc. 81: 1-16.

Flores-Villela, O. 1980. Los reptiles de importancia económica en México. Tesis Profesional. Universidad Nacional Autónoma de México, México.

Guerra-Martínez, V. \& S. Ochoa-Gaona. 2006. Evaluación espacio-temporal de la vegetación y uso del suelo en la Reserva de la Biosfera Pantanos de Centla, Tabasco
(1990-2000). Invest. Geogr. Bol. Inst. Geogr. UNAM 59: 7-25.

Guichard Romero, C.A. 2006. Situación actual de las poblaciones de tortuga blanca (Dermatemys mawii) en el sureste de México. Instituto de Historia Natural y Ecología. Informe final SNIB-CONABIO proyecto No. AS003 México D.F., México.

Graham, T.E. 1979. Life history techniques in turtles, p. 229-243. In M. Harless \& H. Morloock. Turtles: Perspectives and research. Wiley Intercience, Nueva York, EEUU.

INE. 2000. Programa de Manejo. Reserva de la Biosfera Pantanos de Centla. México. Instituto Nacional de Ecología, Secretaría del Medio Amiente Recursos Naturales y Pesca, México, D.F., México.

INE-SEMARNAP. 2000. Balance del Programa Nacional de Áreas Naturales Protegidas. México.

INEGI. 2000. Cuaderno Estadístico municipal de Centla. INEGI, Aguascalientes, Aguacalientes, México.

INIREB. 1986. Plan de manejo para la Reserva de la Biosferas "Los pantanos de Centla, Tabasco". INIREB, Tabasco, México.

Ippi, S. \& V. Flores. 2001. Las tortugas neotropicales y sus áreas de endemismo. Acta Zool. Mex. 84: 49-63.

Iverson, J. \& R. Mittermeier. 1980. Dermatemydidae: River turtles. Catalogue of American Amphibians \& Reptiles 237: 1-4.

Jones, B.K. 1986. Amphibians \& Reptiles p. 267-290. In A.Y. Coperrider, R.J. Boyd \& H.R. Stuards. Inventory and monitoring of wildlife habitat. US Dep. Int. Berou Land Management. Service Center, Denver, Colorado, EEUU.

Joyal, L.A., M. McCollough \& M.L. Hunter. 2001. Landscape ecology approaches to wetland species conservation: a case study of two turtle species in southern Maine. Conserv. Biol. 15: 1755-1762.

Kolbe, J.J. \& F.J. Janzen. 2002. Spatial and temporal dynamics of turtle nest predation: edge effects. Oikos 99: $538-544$.

Lee, R.C. 1969. Observing the tortuga blanca. Int. Turtle Soc. J. 3: 32-34.

Marchand, N. \& J.A. Litvaitis. 2004. Effects of habitat features and landscape composition on the population structure of a common aquatic turtle in a region undergoing rapid development. Conserv. Biol. 18: 758-767. 
Moll, D. 1986a. Food habitats and evidences of spring bank nesting in Belizean Dermatemys mawii. p. 97.

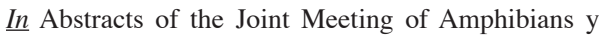
Reptiles and the Herpetologist League. Society for the Study of Amphibians and Reptiles. St. Louis, Missouri, EEUU.

Moll, D. 1986b. The distribution, status, and level of exploitation of the freshwater turtle, Dermatemys mawii in Belize, Central America. Biol. Conserv. 35: 87-96.

Moll, D. 1989. Food and feeding behavior of the turtle, Dermatemys mawii, in Belize. J. Herpetol. 23: 445447.

Moll, E.O. \& J.M. Lagler. 1971. The life history of a neotropical slider turtle, Pseudemys scripta (Schoepff), in Panama. Bull. of the los Angeles Country. Museum of Natural History. Science 11: 1-102.

Moll, D. \& E.O. Moll, 2004. The ecology, exploitation and conservation of river turtles. Oxford University, New York, EEUU.

Morjan, C.L. \& F.J. Janzen. 2003. Nest Temperature Is Not Related to Egg Size in a Turtle with TemperatureDependent Sex Determination. Copeia 2003: 366372.

Novelo R., A. 2006. Plantas Acuáticas de la Reserva de la Biosfera Pantanos de Centla. Espacios Naturales y Desarrollo Sustentable, A.C. México, D.F., México.

Ojasti, J. 2000. Manejo de Fauna Silvestre Neotropical. F. Dallmeier (ed.). SIMAB series No. 5. Smithsonian Institution/MAB Program, Washington, D.C., EEUU.

Plummer, M.V. 1979. Collecting and marking, p. 45-60. In M. Harles \& H. Morloock (eds). Turtle: Perspective and Research. Wiley, Nueva York, EEUU.

Polisar, J. 1990. Exploitation and management of the Central America River Turtle, Dermatemys mawii, in Belize. Tortoise \& Turtles 5: 12-13.

Polisar, J. 1995. River turtle reproductive demography and exploitation patterns in Belize: implication for management. Vida Silv. Neotrop. 4: 10-19.

Polisar, J. 1996. Reproductive biology of a flood-season nesting freshwater turtle of the northern Neotropics: Dermatemys mawii in Belize. Chelonian. Conserv. Biol. 2: 13-25.

Sánchez A.J., M.A. Salcedo, R. Florido, C. Rodríguez, A Galindo \& E. Moguel. 2007. Pantanos de Centla, un humedal costero tropical. In G. de la Lanza-Espino
\& J.L. García-Calderón (eds.). Las aguas interiores de México: Conceptos y Casos. AGT, México, D.F., México.

Segnini, S. \& M. Chacón. 2005. Caracterización fisicoquímica del hábitat interno y ribereño de ríos andinos en la cordillera de Mérida, Venezuela. Sociedad Venezolana de Ecología. Ecotrópicos 18: 38-61.

Semlitsch, R. \& J. Bodie. 2003. Biological criteria for buffer zones around wetlands and riparian habitats for amphibians and reptiles. Conserv. Biol. 17: 12191228.

Tudela, F. 1992. La modernización Forzada del Trópico: el caso de Tabasco. Proyecto integrado del Golfo. Colegio de México-Cinvestav-IFIAS-UNRISD. México, D.F., México.

Turtle Conservation Fund. 2002. A Global Action Plan for Conservation of Tortoises and Freshwater Turtles. Strategy and Funding. Prospectus 2002-2007. Conservation International and Chelonian Research Foundation. Washington, D.C., EEUU.

IUCN 2009. IUCN Red List of Threatened Species. Version 2009.2. (Downloaded: January 19, 2010, www. iucnredlist.org).

Uhmann, T.V., N. Kenkel \& R.K. Baydack. 2001. Development of a Habitat Suitability Index Model for Burrowing Owls in the Eastern Canadian Prairies. J. Raptor. Res. 35: 378-384.

Vayssie'res, M.P., R.E. Plant \& B.H. Allen-Diaz. 2000. Classification trees: An alternative non-parametric approach for predicting species distributions. J. Veg. Sci. 11: 679-694.

Velázquez V.G. 1994. Los recursos hidráulicos del Estado de Tabasco. Ensayo Monográfico. Universidad Juárez Autónoma de Tabasco, Villahermosa, Tabasco, México.

Vogt, R.C. 1980. New method for trapping aquatic turtles. Copeia 1980: 368-371.

Vogt, R.C. 1992. Ecología y estatus de la tortuga blanca (Dermatemys mawii). Reporte Técnico. Consejo Nacional de Ciencia y Tecnología, Universidad Nacional Autónoma de México, México.

Vogt, R.C. \& O.A. Flores-Villela. 1986. Determinación del sexo en Tortugas por la temperatura de incubación de los huevos. Ciencia 37: 21-32.

Vogt, R.C. \& O.A. Flores-Villela. 1992a. Effects of incubation temperature and sex determination in a commu- 
nity of neotropical freshwater turtles in southern of México. Herpetologica 48: 265-270.

Vogt, R.C. \& O.A. Flores-Villela. 1992b. Aspectos de la ecología de la tortuga blanca (Dermatemys mawii) en la Reserva de la Biosfera Montes Azules p. 221-231. In M.A. Vázquez-Sánchez \& M.A. Ramos (eds.). Reserva de la Biosfera Montes Azules: Investigación para su conservación. Ecosfera. San Cristobal de las Casas, Chiapas, México.

Vogt, R.C., G.P. Gonzalez-Porter \& P.P. Van Dijk. 2006. Dermatemys mawii. In IUCN 2009. IUCN Red List of Threatened Species. Version 2009.2. (Downloaded: January 19, 2010, www.iucnredlist.org).
West, R.C., N.P. Psuty \& B.G. Thom. 1985. Las tierras bajas de Tabasco en el sureste de México. Gobierno del Estado de Tabasco. Instituto de Cultura de Tabasco, Villahermosa, Tabasco, México.

Welch, E.B., J.M. Jacoby \& C.W. May. 1998. Stream quality, p.69-94. In E.B Welch, J.M. Jacoby \& C.W. May (eds.). River Ecology and management. Lessons from the Pacific Coastal. Springer, Nueva York, EEUU.

Zenteno R.C. 1993. Estudio de la reproducción de tres especies de tortugas de agua dulce en el estado de Tabasco, México. Tesis de licenciatura, Universidad Juárez Autónoma de Tabasco, Villahermosa, Tabasco, México. 